\title{
Archéopages
}

Archéopages

Archéologie et société

45 | 2017

De la terre au pot

\section{Lezoux, un complexe majeur de production de céramiques durant l'Antiquité}

Synthèse de 40 ans de recherche

Lezoux, a major ceramics production complex during ancient times. An overview of 40 years of research

Lezoux: un complejo mayor de producción de cerámicas durante la Antigüedad. Síntesis de 40 años de investigación

Philippe Bet, Bertrand Dousteyssier et Pierre Pouenat

\section{OpenEdition}

Journals

Édition électronique

URL : https://journals.openedition.org/archeopages/2819

DOI : 10.4000/archeopages. 2819

ISSN : 2269-9872

Éditeur

INRAP - Institut national de recherches archéologiques préventives

Édition imprimée

Date de publication : 1 mars 2018

Pagination : 42-55

ISSN : 1622-8545

Référence électronique

Philippe Bet, Bertrand Dousteyssier et Pierre Pouenat, « Lezoux, un complexe majeur de production de céramiques durant l'Antiquité », Archéopages [En ligne], 45 | 2017, mis en ligne le 01 janvier 2020, consulté le 02 juin 2021. URL : http://journals.openedition.org/archeopages/2819 ; DOI : https:// doi.org/10.4000/archeopages.2819 


\section{Lezoux, un complexe majeur de production de céramiques durant l'Antiquité \\ Présentation de 40 ans de recherche}

Philippe Bet Irrap, UMR S138, «Archóologie et Archóométrie «,

Bertrand Dousteyssier universite Clemmont-Auvergne, USR. 3550

Pierre Pouenat Irrap

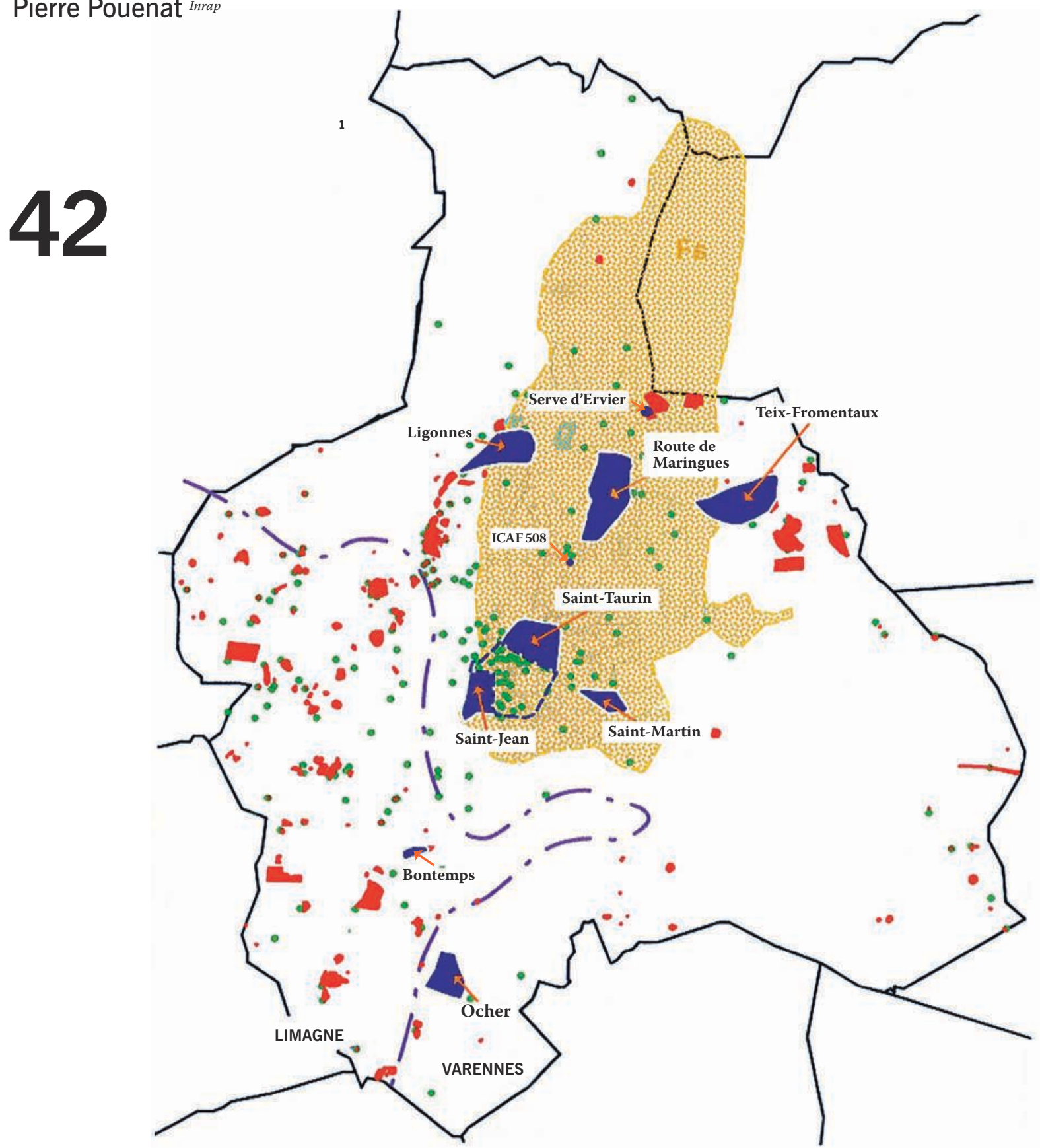

entités recensées avant 1988

entités documentées à la fin du $\mathrm{xx}^{\mathrm{e}}$ siècle

groupes d'ateliers de potiers de l'Antiquité

Sables

$\Lambda^{N}$

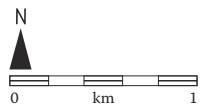

1. Carte archéologique de Lezoux. Les ateliers de potiers de l'Antiquité sont principalement implantés sur les sables clairs de la formation géologique $F$ dans le secteur des Varennes. 
À mi-chemin entre Clermont-Ferrand et Thiers, la petite ville de Lezoux est dotée d'un grand musée départemental, créé il y a dix ans, qui reflète le lien étroit entretenu par ce lieu avec la céramique depuis plus de deux millénaires. Le témoin le plus ancien a été découvert en 1987 sur la ZAC de l'Enclos, près de l'hôtel de ville. Il s'agit d'un four isolé, daté de La Tène Finale (Mennessier-Jouannet, 1991). La production de poterie durant la période gauloise a vraisemblablement été plus importante que ce que l'archéologie nous a livré jusquà présent ${ }^{1}$. Quoi qu'il en soit, c'est la période romaine qui a donné, de façon spectaculaire, ses lettres de noblesse à Lezoux. Dès la fin de la période augustéenne, un artisanat céramique très important est déjà bien attesté. Seuls les artisans œuvrant autour de la rue Saint-Taurin sont connus par leur nom, parce qu'ils fabriquent une poterie particulière : la sigillé $e^{2}$. Certaines formes nécessitent obligatoirement la présence d'un timbre d'un type particulier sur leur fond intérieur et cette marque fait partie intégrante de la forme (Bet, Delor, 20oo). C'est le cas systématiquement des assiettes, des plats, ainsi que de la plupart des formes de coupelles. Un grand nombre de céramistes, restés anonymes, secondés par des ouvriers et des apprentis, fabriquaient d'autres catégories de poteries, fines ou communes. L'extraction et la préparation de l'argile, la collecte de bois nécessitaient également de la main-d'œuvre. Outre le groupe d'ateliers de la rue Saint-Taurin, d'autres lieux étaient voués à la production céramique dès les premières années $\mathrm{du} \mathrm{I}^{\mathrm{er}}$ siècle. Plus au nord, à quelques milliers de mètres de distance, les ateliers de la route de Maringues et ceux de Ligonnes étaient également actifs mais ne fabriquaient pas encore de sigillée. Centre de production majeur dès la fin du règne d'Auguste, Lezoux impose sa suprématie dans la moitié nord de l'Empire romain au II $^{\mathrm{e}}$ siècle (Bet, Vertet, 1986).

\section{Le choix du site}

La raison principale de l'implantation de ces officines de potiers à Lezoux ne nous est pas connue. Évidemment, une argile de qualité se trouve dans son sous-sol, mais celle-ci est

1. Des fouilles

préventives, à l'initiative d'Hugues Vertet

(Bet, Dousteyssier, 2014, p. 15-17; Bet, 2015 ; Bet, 2016), ont été menées dès le tout début des années 1960 avec des bénévoles. Il faudra attendre 2011 pour une meilleure prise en compte du patrimoine et une normalisation progressive des procédures.

2. Cette céramique au vernis rouge brillant doit son nom à la signature du potier fréquemment estampée sur celle-ci (sigillum signifie sceau, en latin). déposée anciennement par la Dore (Bet, 2012) [ill. 1]. Cette zone de contact offrait un accès aisé aux ressources essentielles (argile, bois et eau). Si l'argile est, pour ainsi dire, inépuisable à l'échelle de cette activité, la quantité de bois nécessaire aux cuissons durant tout le Haut-Empire a dû être considérable. La cuisson des sigillées grésées cuites aux environs de $1000{ }^{\circ} \mathrm{C}$ par rayonnement à travers des tubulures qui canalisaient gaz et fumées nécessitait davantage de combustible que celle des poteries communes ou des céramiques fines cuites en flammes directes. L'eau est abondante, la nappe phréatique peu profonde, et la densité des puits et des réseaux d'adduction la rendait facilement accessible.

Les officines céramiques de Lezoux n'étaient pas destinées à desservir un marché régional. Durant le $\mathrm{II}^{\mathrm{e}}$ siècle, la diffusion de la sigillée est à son apogée et s'étend, par un commerce régulier, sur toute la moitié septentrionale de l'Empire romain, soit sur plus d'un million de kilomètres carrés. À environ 7 km des ateliers, l'Allier, alors navigable, a dû jouer un rôle considérable dans l'exportation des productions lezoviennes. Cette rivière, à partir de sa confluence avec la Loire, permettait une large diffusion à travers l'axe ligérien et ouvrait les marchés de la Bretagne romaine. Ainsi, l'utilisation des voies fluviales et maritimes complétait les possibilités d'exportation par la route à partir du grand axe Saintes-Lyon qui passait à proximité [ill. 2] .

La question de la propriété des terrains sur lesquels sont bâtis les ateliers reste posée. Dépendaient-ils de villce de Limagne? Appartenaient-ils à de grands propriétaires gaulois, à des citoyens romains, à des negociatores, aux potiers eux-mêmes ? Ces terrains ont-ils été loués aux artisans, ce qui aurait favorisé leur mobilité ? On retrouve certains potiers de Lezoux ailleurs en Gaule ou en Germanie. Faute d'avoir la preuve d'un artisanat potier conséquent à l'époque gauloise, on se demande quelle a été la raison de la venue de tous ces céramistes à Lezoux à la fin du règne d'Auguste. Le succès de quelques précurseurs est-il suffisant pour expliquer, en quelques années, le regroupement de tant de potiers ? S'agissait-il de démarches individuelles ou de l'action de quelques commanditaires? Le regretté professeur Jan Kees Haalebos avait même voulu voir une main impériale derrière la création de quelques grands centres de céramique sigillée (Haalebos, 1977), à l’instar des manufactures de tuiles en Italie (Jouanaud, 1994). Cette hypothèse induit que la sigillée aurait été, en quelque sorte, une céramique de propagande, mais elle n'a pu être démontrée.

\section{L'organisation des ateliers et le dynamisme de production}

Les officines céramiques occupent plus de 90 ha avec des ensembles importants d'une superficie variant entre 12 et 21 ha. Ces groupes d'ateliers de un rôle. À l'ouest s'étend la plaine de la Grande Limagne, de bonnes terres fertiles, où l'argile verte de l'oligocène se trouve à faible profondeur. De nombreuses grandes villee (Dousteyssier et al., 2004) ont exploité tout ce secteur et leurs vestiges incitent à penser que l'opulence devait y régner À l'est, c'est tout l'inverse. Ce sont sur ces premiers terrains peu propices à l'agriculture que les ateliers antiques de Lezoux se sont installés, essentiellement sur une nappe alluviale sableuse
\% 


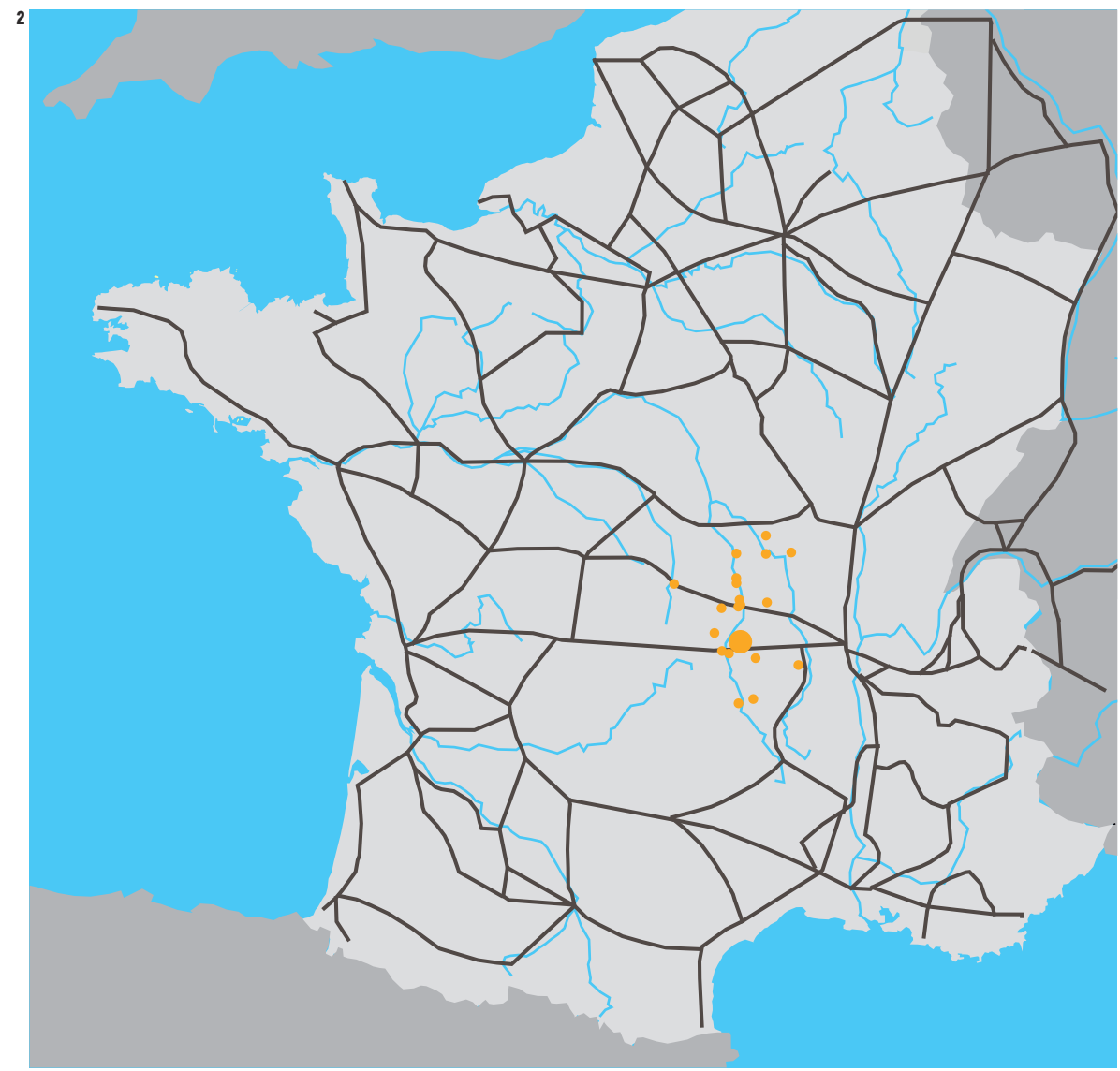

3. Les principaux centres

de production de sigillée

dans la partie nord-ouest

de l'Empire romain entre

la seconde moitié du Ier $^{\text {siècle }}$

avant notre ère et le ve siècle.

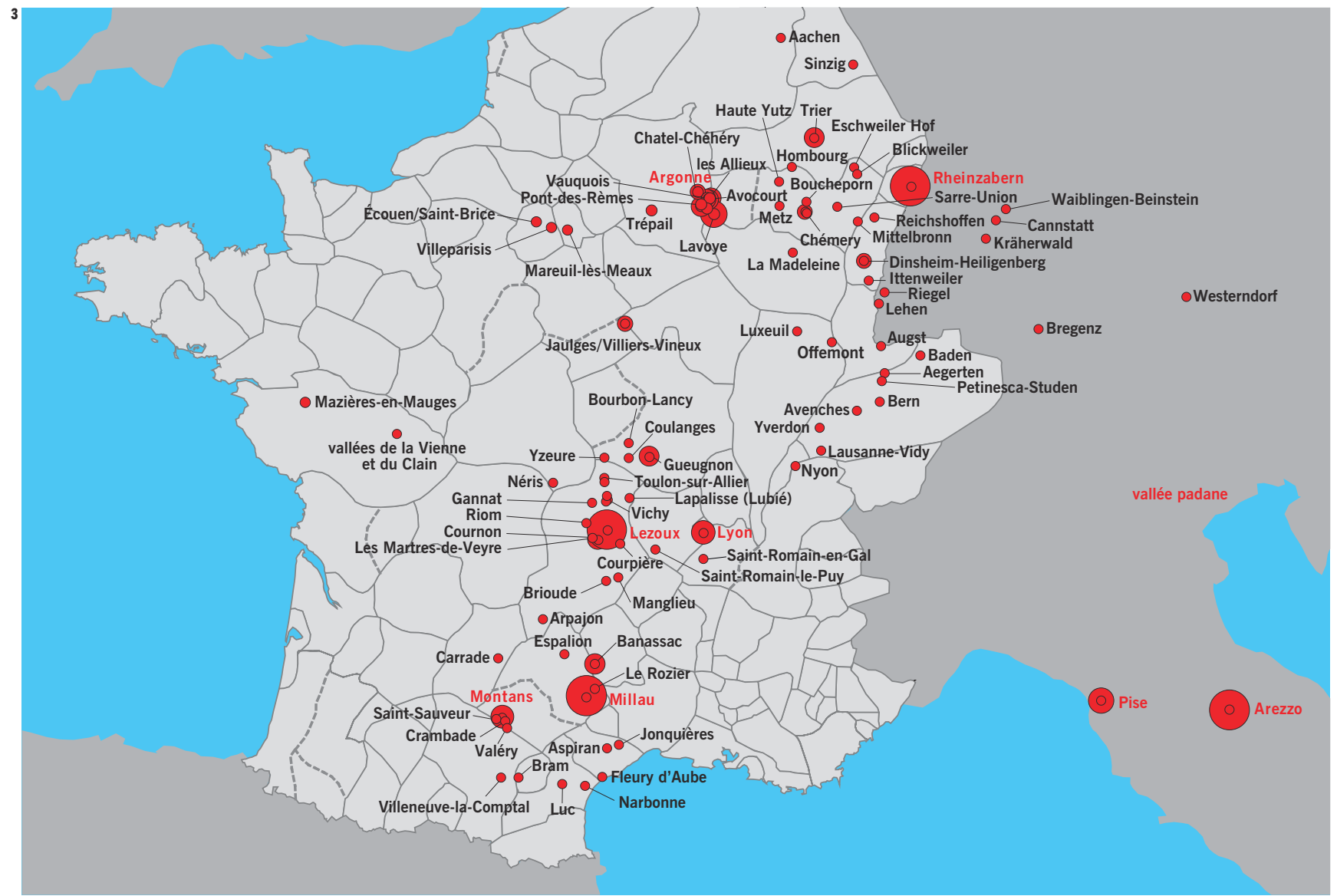

- voies principales

- Lezoux et ateliers de la Gaule centrale

— limites de cité

-- $\quad$ subdivision de cités du Bas-Empire

lieux de production de sigillée

Lezoux lieux de production les plus importants

$\Lambda^{N}$

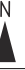


potiers réunissaient les unités de tournage et de cuisson, mais également l'habitat, des sanctuaires et des lieux funéraires (Bet, 1988). Quelques officines paraissent cependant isolées. C'est le cas de celle, datée du II $^{\mathrm{e}}$ siècle, de la Serve d'Ervier (Henry, 2014, p. 74-75) au nord des ateliers de la route de Maringues et également du site «ICAF 508 » qui apparaît comme une implantation tardive $\mathrm{du} \mathrm{III}^{\mathrm{e}}$ siècle entre les ensembles de Saint-Taurin et de la route de Maringues. Au tout début du $\mathrm{I}^{\mathrm{er}}$ siècle de notre ère, seuls trois groupes sont attestés : ceux de Ligonnes (Chuniaud, 2002), de la route de Maringues et, enfin, celui de SaintTaurin. Ce dernier apparaît comme le noyau dur de la production, avec une activité attestée à toutes les époques et des productions très variées ; la fabrication de la sigillée est initiée en ce lieu et elle s'y poursuit jusqu'au Bas-Empire. Les deux autres groupes d'ateliers semblent n'avoir fonctionné que durant le Haut-Empire et n'ont quasiment pas produit de sigillée pendant tout le $\mathrm{I}^{\mathrm{er}}$ siècle, mais plusieurs catégories de céramiques fines (à engobe blanc ou rouge, terra nigra...). À partir de la seconde moitié du siècle, les parois fines engobées y sont fabriquées en masse. Ce n'est qu'au tout début du $\mathrm{II}^{\mathrm{e}}$ siècle que ces productions déclinent au profit de la sigillée, ce qui demande de remplacer les anciens fours par des fours à tubulures et d'employer un nouveau type d'argile. Pour assurer le tournage de centaines de millions de vases, les trois groupes d'ateliers initiaux ne sont plus suffisants. De nouvelles officines se regroupent alors en d'autres lieux : à Ocher, aux Saints-Jean, au Theix-Fromentaux (Ferdière, 2014, p. 204-205), aux Bontemps, à Saint-Martin. Elles resteront actives durant plusieurs décennies. Cet essor bénéficie également à toute la région située entre Issoire et Nevers. De nouveaux ateliers voient le jour à Courpière/Bellîmes (Bet, Valaude, Vertet, 1986), Cournon/Les Queyriaux (Bet, Boudriot et al., 1998), Bellerive-sur-Allier/TerreFranche (Vauthey, 1993), Gannat (Vertet, 1980), Gueugnon (Notet, 2012), Manglieu (Bet, Delage, Murat, 200o), Lapalisse (Corrocher, 1994), Les Martres-de-Veyre (Romeuf, 200o), Toulon-surAllier (Féraudy, Vertet, 1986)... Ils partagent tous des traits communs avec Lezoux et un style du Centre de la Gaule s'affirme nettement, bien différent de celui du Sud (Rogers, 1974 et 1999; Stanfield, Simpson, 1990). Des moules de Lezoux sont parfois acquis par ces ateliers, mais d'autres sont également conçus et fabriqués sur place. Ces « ateliers de potiers de la Gaule centrale » (Vertet, 1980) sont principalement implantés en terre arverne, mais quelques-uns se trouvent en bordure de celle-ci, sur le territoire des Bituriges Cubes ou dans la puissante cité éduenne. Les normes techniques, morphologiques et qualitatives rigoureusement respectées à Lezoux sont plus ou moins appliquées dans les centres secondaires de production. Ceux-ci diffèrent aussi de Lezoux par leur superficie réduite. Elle se mesure généralement en milliers de mètres carrés. Seuls les ateliers éduens de Gueugnon (15 à 20 ha), en Saône-et-Loire, et Coulanges (4 ha), spécialisé surtout dans la fabrication de mortiers en terre blanche, présentent des emprises un peu plus vastes. Vichy pourrait également occuper une surface se chiffrant en hectares. Au II $^{\mathrm{e}}$ siècle, la diffusion de la sigillée de Lezoux et du Centre de la Gaule est à son apogée [ill. 3].

\section{Les carrières et la préparation de l'argile}

Pour le groupe des ateliers de la route de Maringues, la limite nord-ouest est extrêmement rectiligne et reste bien marquée dans le paysage actuel. Comme tendraient à le démontrer de nombreux carottages archéologiques effectués à l'ouest de cette limite longue de plus de $300 \mathrm{~m}$, nous serions ici en présence du front de la carrière où les potiers du secteur s'approvisionnaient en argile. Cette zone d'extraction, en contrebas des ateliers, a été ensuite remblayée avec les refus de terre auxquels se mêlaient des débris céramiques et des ratés de cuisson. Une autre vaste carrière a été découverte à $2300 \mathrm{~m}$ à l'ouest du groupe de la rue Saint-Taurin ; sondages et carottages ont montré qu'elle a été comblée sur plusieurs mètres de la même façon que la précédente. Située au Pont-desMoulins, en limite communale de Lezoux, elle se trouve en bordure d'un gros ruisseau, le Litroux, et de l'ancienne route nationale reliant Bordeaux/ Saintes à Lyon. Il est possible que, durant l'Antiquité, l'examen des rives de celui-ci ait amené l'exploitation de ce secteur. Les multiples fossés de drainage, qui ont permis d'assécher les marais de la Limagne depuis l'époque gauloise, ont nécessairement apporté une connaissance du soussol et mis en évidence les argiles sous-jacentes.

Dès le $\mathrm{II}^{\mathrm{e}}$ siècle, afin de disposer de la matière première en quantité pour la fabrication de la sigillée, de grandes aires de préparation sont construites en bordure nord-est des ateliers de la rue Saint-Taurin (Vertet, Bet, 1980). Elles devaient approvisionner l'ensemble des officines du secteur [ill. 4]. Durant la seconde moitié du IV ${ }^{\mathrm{e}}$ siècle, aux mêmes emplacements et en respectant les mêmes axes, des installations similaires se maintiennent pour fournir l'argile nécessaire aux dernières sigillées arvernes [ill. 5]. Plus au nord, dans le groupe des ateliers de la route de Maringues, il semble que chaque officine disposait de son aire de préparation. La plus grande, découverte sur le terrain Dardinier en 2011 (Bet, Delage, Pouenat, 2012), a une superficie d'environ $50 \mathrm{~m}^{2}$ [ill. 6] et les plus petites devaient avoisiner une vingtaine de mètres carrés ${ }^{3}$. Les aménagements d'aires de préparation de l'argile ne sont pas fréquemment retrouvés en contexte archéologique, malgré de nombreuses fouilles d'ateliers. Celles qui ont été retrouvées à Gueugnon (Saône-et-Loire) et à Rheinzabern (Rhénanie-Palatinat) pourraient ainsi être liées à l'influence de potiers lezoviens ayant migré dans ces régions. 

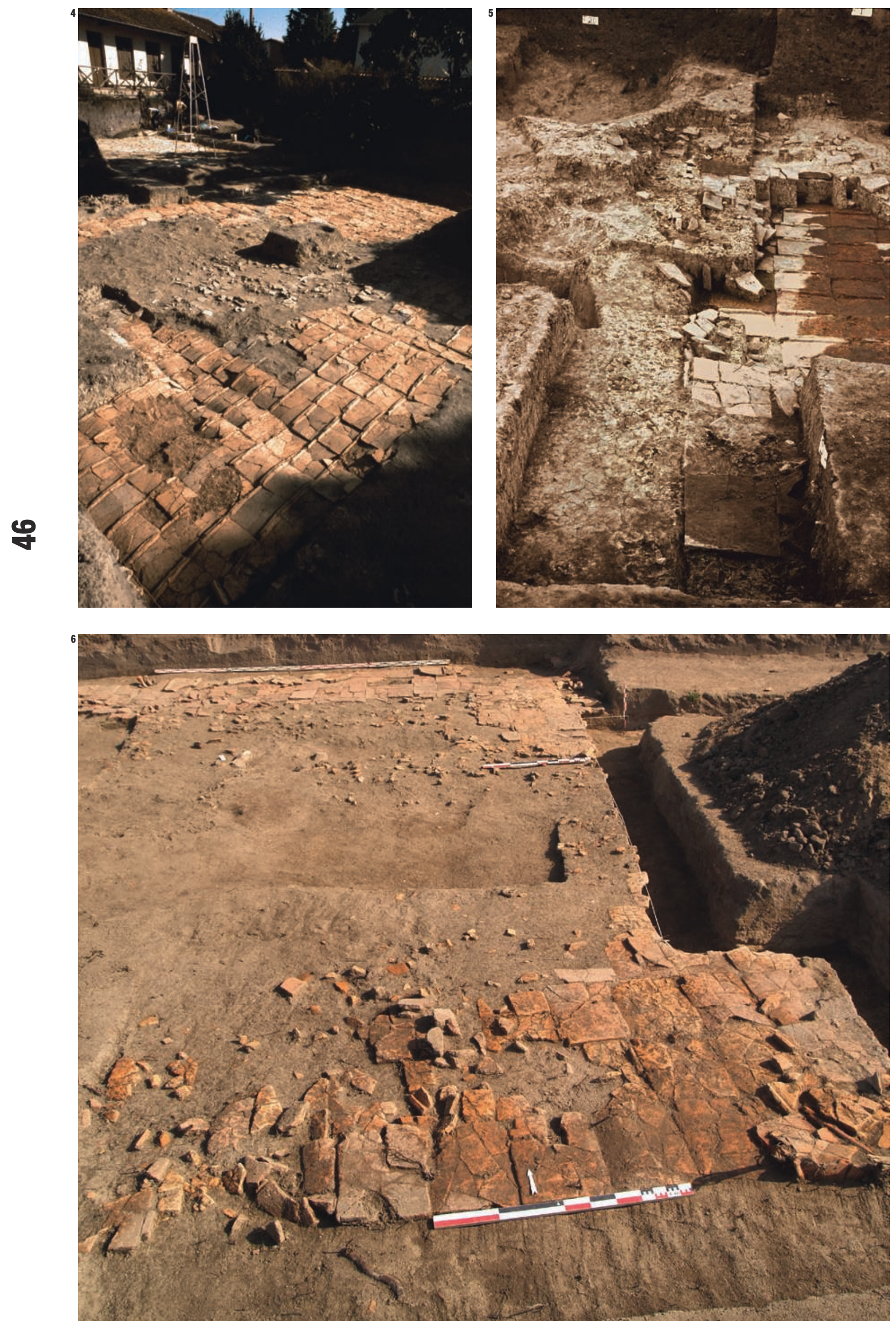

4. Les aires de préparation de l'argile, dans le groupe des ateliers de la rue Saint-Taurin, ont été fouillées entre 1977 et 1979 (site de l'CEuvre Grancher). D'une superficie individuelle de $120 \mathrm{~m}^{2}$, elles présentaient un fond dallé avec environ un millier de tuiles à rebords. Ce type d'installation est inconnu dans les ateliers du sud de la Gaule et a dû être mis au point à Lezoux au ॥t siècle, au moment où les potiers doivent faire face à une énorme demande du marché. 5. Dans la partie nord-ouest du chantier de l'CEuvre Grancher, en 1977, a été mise au jour, à une trentaine de centimètres au-dessus des installations du Haut-Empire, une aire de préparation de l'argile de la seconde moitié du Ive siècle. Son fond était recouvert par des dalles rectangulaires en terre cuite. D'une superficie d'environ $50 \mathrm{~m}^{2}$, sa limite occidentale et son orientation étaient rigoureusement identiques à celle précédemment installée au II siècle. Celle-ci était également parallèle au mu de parcelle construit au xixe siècle, montrant une certaine permanence dans

les axes d'implantation depuis l'Antiquité. Tout le secteur était recouvert par une épaisse couche d'argile jaune salie que perforaient des installations du Moyen Âge.

6. Dans le groupe des ateliers de la route de Maringues, les aires de préparation de l'argile sont de taille plus réduite que celles des ateliers de la rue Saint-Taurin. La partie centrale de ces installations est souvent assez endommagée, en raison sans doute d'un travail plus fourni à cet emplacement. Ici, sur le terrain Dardinier, en 2011. la limite orientale de l'aire a été perforée par le creusement d'une tranchée de fondation d'un pavillon. 
Les premières sigillées dans la diversité des productions du $\mathrm{I}^{\mathrm{er}}$ siècle

La fabrication de la sigillée est attestée à Lezoux dès la dernière décennie du règne d'Auguste. Une estampille circulaire, sur une coupelle en sigillée, nous présente la tête d'un jeune homme de profil avec la mention TIBER CAESAR (Bet, Dousteyssier, 2014, p. 34-35). Il s'agit d'une marque commémorative saluant l'accession du prince impérial au titre de César du vivant de l'empereur Auguste. Le démarrage des ateliers de sigillée en ce tout début du $\mathrm{I}^{\text {er }}$ siècle est fulgurant (Vertet, 1967). Plus de 120 producteurs et décorateurs de sigillée sont connus durant cette phase initiale (Bet, 1988) qui dura probablement relativement peu de temps. Comme le pressentait Jean-Jacques Hatt depuis longtemps, l'influence arrétine est, curieusement, bien plus forte à Lezoux que celle des succursales lyonnaises distantes de seulement $150 \mathrm{~km}$. Les liens avec les officines italiques d'Arezzo sont parfois affichés de façon ostensible sur les marques qui poinçonnent les vases. Des potiers, comme Rutenos, Flavus, Nasios ou Duratus par exemple, font suivre leur nom d'une mention abrégée de FECIT ARETINVM signifiant qu'ils travaillaient à la façon arrétine. D'autres portent des noms qui rappellent singulièrement les grands potiers arrétins qui ont établi la notoriété, depuis l'Étrurie, de cette céramique sigillée. Ainsi, Perenias a-t-il peut-être été un ouvrier de Perrenius, l'intarissable décorateur d'Arezzo [ill. 7]?

Pour obtenir des vases ornés ou figurés, les potiers avaient recours à l'emploi d'un moule dans lequel ils réalisaient un décor en creux [ill. 8]. Ils utilisaient la propriété qu'a l'argile humide de se rétracter d'environ $10 \%$ au séchage. Pour réaliser le moule, ils tournaient une forma correspondant à la partie décorée du vase. Celle-ci devait avoir un angle d'ouverture suffisant pour permettre, après rétraction de la terre, le dégagement sans effort du vase. Après un prétraçage souvent fait légèrement au stylet, le décor était estampé avec des poinçonsmatrices dans une argile maintenue humide. Après séchage et cuisson, le moule devait rester suffisamment poreux pour faciliter le démoulage. Ensuite, il était fixé au tour et le potier tournait à partir de celui-ci l'intégralité du vase jusqu'à la lèvre. Une vingtaine d'heures de séchage était nécessaire pour permettre d'extraire sans difficulté le vase de son moule. Chaque tourneur devait donc disposer d'un grand nombre de moules pour

4. C'est la fouille d'établissements militaires romains datés historiquement, sur le limes de l'Empire, qui a permis de construire une chronologie relativement fine de la sigillée.

5. Suprématie partagée avec les ateliers de

Montans (Tarn) pour

la façade atlantique de

la France et le nord-ouest de l'Espagne. produire plusieurs vases en une journée de travail fabrication des vases décorés, ainsi que les poinçons-matrices, sont largement inspirés de ceux d'Aretium. Les mêmes scènes sont retranscrites. Des poinçons figurés sont façonnés à Lezoux, mais il ne s'agit pas de surmoulage. Ce sont visiblement des outils qui ont été refaits sur place d'après le souvenir de ceux que les potiers lezoviens avaient utilisés en Italie. Chaque modèle garde la même expression, la même attitude. Ici, un bûcheron est réalisé avec deux poinçons distincts, permettant de faire figurer ou non sa jambe gauche, ou de la placer différemment. Ailleurs, un satyre dansant peut être représenté avec différents accessoires qu'il tient entre ses deux mains et qui sont tracés à la demande avec un stylet (baguette...) ou poinçonnés (feuille...). En revanche, les poinçons de Lezoux, même s'ils sont de qualité honorable et avec une plastique certaine, n'atteignent pas la quasi-perfection du modèle original. La pardalide de ce satyre est devenue presque méconnaissable, ressemblant davantage à un drapé qu'à une peau de panthère, et sa chevelure est devenue crépue (Bet, Montineri, 1989). En ce qui concerne les formes des vases, elles semblent respecter en tous points le « cahier des charges » suivi à Arezzo. Le moindre détail morphologique est présent et le mode d'estampillage scrupuleusement suivi. Il y a cependant des différences dans les espaces de travail entre les deux grands centres. Alors qu'à Arezzo les moules sont constellés de taches rouges montrant que la phase d'engobage était réalisée à proximité de leur lieu de stockage et donc de leur lieu d'utilisation, cela n'est jamais le cas à Lezoux, à une seule exception près, toutes périodes confondues.

Il ne faudrait pas considérer la sigillée comme une céramique de luxe, sauf peut-être à ses débuts et seulement dans des campagnes reculées (Baratte, Joly, Béal, 2007). Elle est largement diffusée dans tout le monde romain et, notamment, dans les camps et forteresses des légionnaires établis sur les frontières ${ }^{4}$. Dans le dernier quart du $\mathrm{I}^{\mathrm{er}}$ siècle avant notre ère, de véritables succursales s'implantent dans la colonie romaine de Lyon avec la venue d'artisans et d'esclaves (Desbat et al., 1996), et il est souvent difficile de distinguer les fabrications gauloises des productions italiques. Ces ateliers ne fonctionnèrent que quelques décennies, mais d'autres lieux, comme Millau (Genin, Schaad, 2007) et Lezoux, s'étaient déjà mis à produire de la sigillée, précipitant peut-être l'arrêt des fours lyonnais.

Les premières productions de sigillée de Lezoux étaient de qualité, mais elles ne réussirent pas à s'imposer (Bet, Delor, 2002). Elles présentaient une différence fondamentale avec les sigillées italiques : les potiers lezoviens n'employaient pas de l'argile calcaire, mais siliceuse et micacée (Picon, 1973 et 2002). Ils ne pouvaient donc pas forcer la température des fours et dépasser les $1000{ }^{\circ} \mathrm{C}$ qui auraient permis la vitrification du vernis. Bien que de bel aspect, l'engobe des vases restait poreux et s'altérait plus vite. Vers la même époque, les officines de Millau, elles, font ce choix d'une argile calcaire, à l'instar des ateliers italiques, choix qui sera à l'origine de la suprématie ${ }^{\mathbf{5}}$, durant presque un siècle, de la vaisselle sigillée dans tout l'Empire romain. L'argile calcaire était pourtant affleurante à proximité des ateliers de Lezoux et les outils et la 

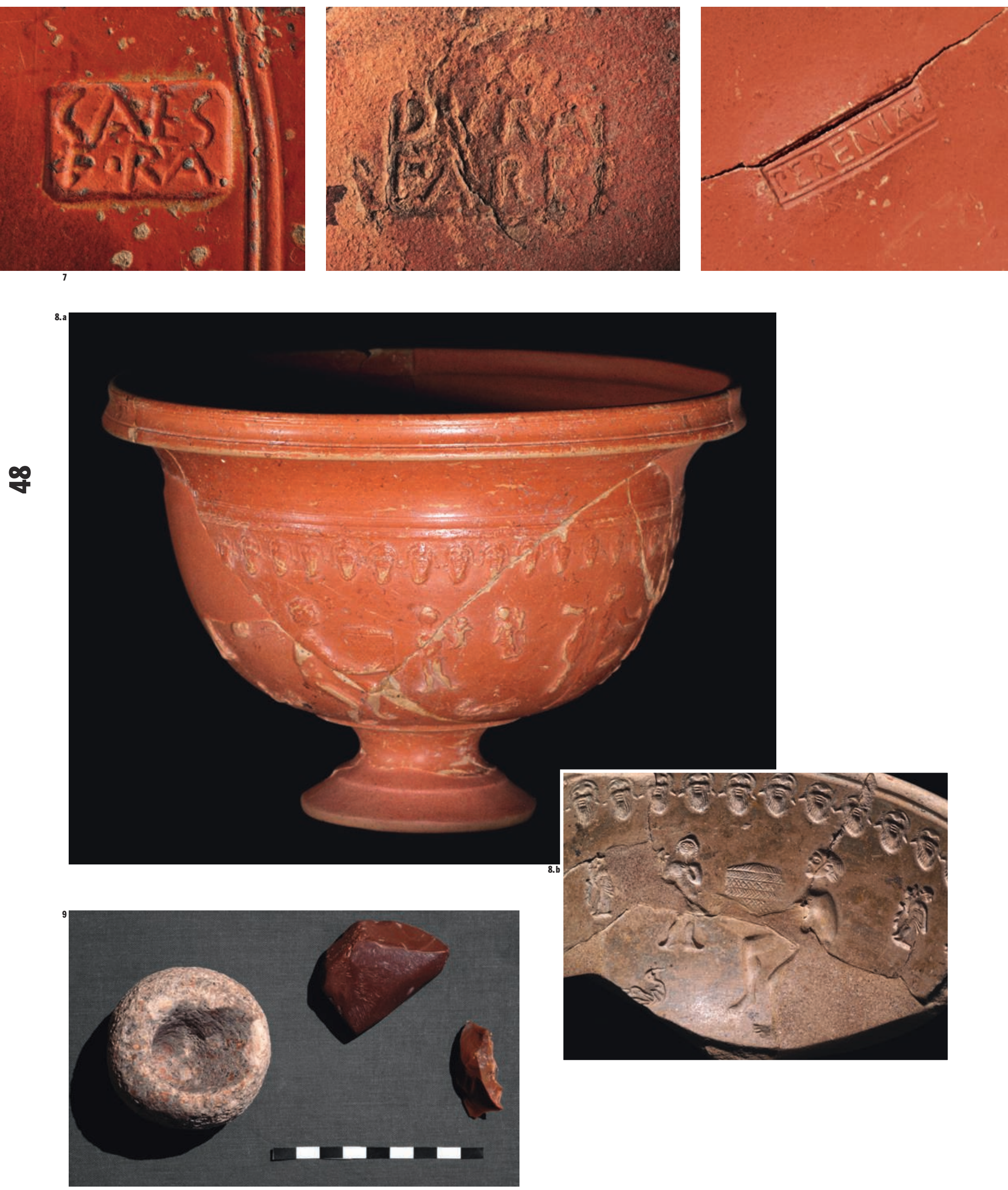
7. Estampilles des potiers de Lezoux. L'inscription CAES F. RA fait sans doute allusion au nouveau César Tỉbère (CAES) et peut-être à Arezzo (RA); le F signifie fecit (a fait [ce vase]). Le potier Duratus a inscrit les quatre premières lettres de son cognomen et FARE pour f[ecit] are[tinum]. Le nom Perenias évoque quant à lui celui du maître potier Perennius d'Arrezo.

8. a. Calice Drag.11 du début du $I^{\text {er }}$ siècle découvert par Hugues Vertet. II représente une scène égyptienne avec la jeune divinité Harpocrate qui invite au silence en dressant l'un de ses doigts devant sa bouche. La frise d'oves qui, généralement, couronne le décor est remplacée ici par des têtes de silène.

b. Moule du calice. À cette époque les poinçons sont faiblement enfoncés dans la matrice, ce qui permettait de démouler plus facilement sans endommager les reliefs par frottement. L'oiseau est à l'origine un motif végétal. Le décorateur l'a transformé en traçant, au stylet, le bec et les pattes.

9. Outils de lissage et crapaudine extrêmement usée d'un tour de potier (site de Lubié à Lapalisse, Allier, II 'e siècle). Dans les ateliers de Gaule centrale, celle-ci est en fait un poids de référence qui a été détourné de son usage premier. technologie permettaient d'obtenir des sigillées à vernis grésé. Au début du $\mathrm{I}^{\mathrm{er}}$ siècle, le four à sigillée le plus important de Lezoux avait un laboratoire de cuisson carré de $2 \mathrm{~m}$ de côté et un alandier de même longueur (Bet, Gangloff, 1987). Ce four aurait pu supporter des températures nécessaires à l'obtention d'un grésage et permettait, déjà, la cuisson des vases en mode protégé, non grâce à l'emploi de gazettes mais avec des tubulures (Desbat, 1993). Quoi qu'il en soit, bien qu'une production de sigillée se maintint durant tout le $\mathrm{I}^{\text {er }}$ siècle, il est très probable que nombre de potiers quittèrent Lezoux durant le règne de Tibère pour rejoindre les ateliers florissants du Sud. C'est sans doute le cas de Rutenos qui a été l'un des décorateurs les plus fameux du centre lezovien. Son nom interpelle, puisqu'il indique une origine rutène, c'est à dire de la région où se trouve Millau. Est-il alors reparti vers sa terre natale au moment de la récession des ateliers arvernes? Le poinçon d'un de ses motifs, d'inspiration arrétine, qui représente un vendangeur a d'ailleurs été retrouvé à Millau. L'analyse physico-chimique a confirmé l'attribution visuelle : il provient bien de Lezoux (Bet, Delage, Vernhet, 1994).

Cet échec de Lezoux dans la fabrication de masse de la sigillée favorisa vraisemblablement le développement de la production de poteries communes, mais surtout d'autres catégories de céramiques fines (Bet, Gangloff, Vertet, 1987). Pour les premières, il s'agit essentiellement de communes claires utilitaires. De différents modules, les formes fabriquées correspondent à des ollce (urnes) à lèvre en bourrelet, des marmites tripodes à bord en collerette avec des pieds creux, des jattes, des cruches et de grands récipients de stockage de type dolium d'au moins $80 \mathrm{~cm}$ de haut et d'un diamètre équivalent. Ces formes évoluent peu entre le milieu du $\mathrm{I}^{\mathrm{er}}$ siècle et le $\mathrm{II}^{\mathrm{e}}$ siècle (Béranger et al., 2015 ; Batigne-Vallet et al., 2016). Une autre catégorie de céramiques communes se caractérise par un engobage rouge, une surface grumeleuse et une pâte peu réoxydée en phase de post-cuisson. Les formes se résument essentiellement à des amphorettes, à bord en entonnoir, munies d'anses plates et larges. Ces poteries communes, engobées ou non, se retrouvent sur une bonne partie du territoire arverne.

Les céramiques fines regroupent de multiples catégories et sont fabriquées à partir d'argiles proches de celles employées pour les sigillées du $\mathrm{I}^{\mathrm{er}}$ siècle. Dès la période augustéenne, les céramiques à engobe blanc sont fabriquées en très grandes quantités dans les trois groupes d'ateliers alors actifs. Il s'agit essentiellement de cruches, mais aussi d'amphorettes. Les récipients plus précoces sont davantage moulurés (anses et cols). La fabrication se simplifie ensuite. Les ollce, les vases-balustres et les biberons, quoique présents dans le répertoire, restent très rares. La production s'achève définitivement durant la première moitié du $\mathrm{II}^{\mathrm{e}}$ siècle.
La terra nigra est produite dans les mêmes ateliers (Bet, Chuniaud, 1995). La surface en est très soigneusement lissée [ill. 9]. Le répertoire typologique comporte quelques dizaines de formes. La plus fréquente, à Lezoux, est une coupe au fond ombiliqué (Bet, 1994, forme 369; Menez, 1989, forme $57 \mathrm{C}$ ). La production massive d'assiettes en sigillée a dû avoir pour répercussion une fabrication moindre dans cette catégorie céramique. En ce qui concerne la terra rubra, avec un engobe qui ne couvre que la face visible du vase, elle ne se rencontre qu'à la période augustéenne. Il est vrai que deux formes de coupes en sigillée (l'une lisse, l'autre moulée) de la période augustotibérienne pourraient s'inscrire dans cette tradition, avec un engobage effectué uniquement sur la paroi extérieure.

À partir de la seconde moitié du I ${ }^{\mathrm{er}}$ siècle, Lezoux se mit à produire, intensivement, des parois fines engobées (Bet, Gras, 1999) jusqu'au milieu du siècle suivant. Il s'agit avant tout de gobelets ovoïdes à lèvre plate, mais il y a également des coupelles apodes, des petits tripodes et des couvercles. L'engobe est souvent de couleur noir à brun, mais il peut aussi être orangé, par ré-oxydation de l'atmosphère du four en phase de refroidissement. Les parois sont décorées ou lisses, surtout pour les petits modules. Comme il s'agit de gobelets à boire, la décoration permet d'augmenter la tenue en main de l'objet. Ainsi, les potiers peuvent rajouter, avec de la barbotine, un décor d'épingles à cheveux ou de lunules ou appliquer des petits grains d'argile pour obtenir une surface granitée. Ils peuvent, également, avec une lame vibrante, faire un guillochis partiel ou total, surtout pour de grands modules. Pour ceux-ci, ils peuvent aussi rajouter des anses et/ou des décors de relief d'applique figurant des personnages ou des animaux (Tourgon, 2014, p. 86-87).

En plus de céramiques fines et communes, les ateliers de Lezoux fabriquèrent également des tuiles, mais aussi des terres cuites architecturales décoratives. Celles-ci étaient revêtues d'un engobe blanc. Les éléments produits correspondent à des colonnettes à chapiteau, des corniches moulurées, des balustrades, des masques... (Pouenat, 2014, p. 76-77).

\section{La suprématie de la sigillée de Lezoux}

Au tout début du règne de Néron, Titos (Piboule et al., 1981), un potier venant de Millau, s'installe à Lezoux. Son arrivée marque le renouvellement du répertoire décoratif et, peut-être, typologique. L'influence de La Graufesenque marque, alors, les productions lezoviennes. Un autre potierdécorateur venant également des ateliers du Sud, Drutanus, fait une halte à Lezoux avant de prendre le chemin de la Lorraine où il maintient son style très reconnaissable (Lutz, 1977). Un peu plus tard, durant la période flavienne, de nouveaux décorateurs s'imposent. Des fabricants de poinçons-matrices de talent revisitent le répertoire 

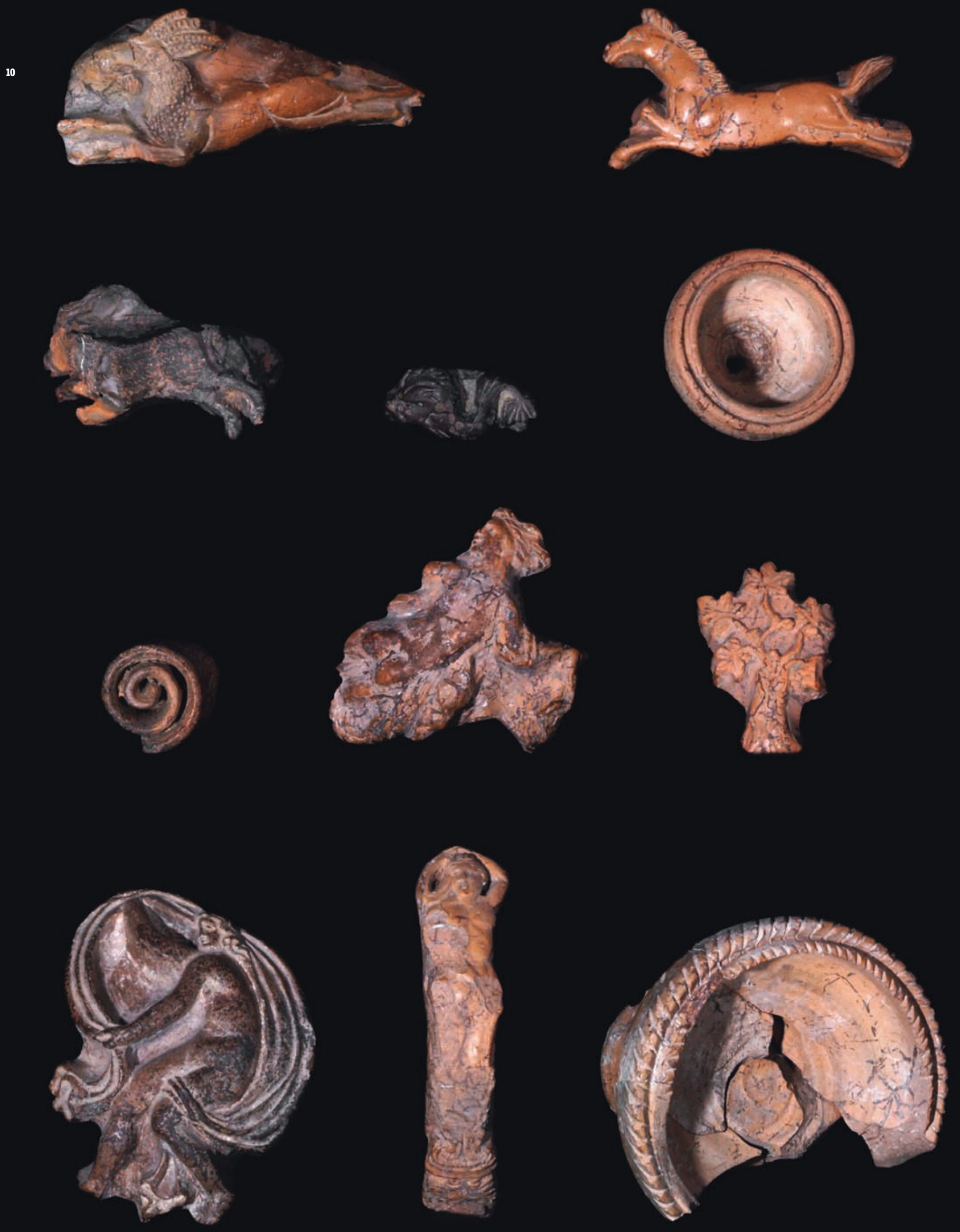
10. Série de poinçonsmatrices du III' siècle

découverts dans un bâtiment incendié de la ZAC de l'Enclos (groupe des ateliers de la rue

Saint-Taurin). Chaque poinçon possède une courbure pour

s'adapter au profil du moule sur lequel il sera employé.

11. Moules de Lezoux

a. Moule de l'officine de

Libertus (groupe des ateliers de la route de Maringues)

du premier quart du II siècle.

Les moules des coupes

hémisphériques Drag. 37

de l'époque flavienne et du

règne de Trajan comportaient

une réserve dans le fond

qui devait être retravaillé,

après démoulage, pour

dégager et façonner le pied.

b. Moule du décorateur

Doeccus de la seconde moitie

du II siècle. Le style est très

classique et représentatif de

l'ornementation lezovienne.

Le décor à métopes figure

les plaisirs du soldat.

Celui-ci est représenté

à quatre reprises dans un

médaillon circulaire.

Il est entouré de divers

personnages (gladiateurs

affrontés, danseuse

au tambourin, femmes).

Le décorateur a placé,

verticalement, son nom

(DOIICCI), décliné au génitif,

en grandes lettres. Le fond

du moule ne comporte pas

de réserve pour le pied.

Après démoulage, ill sera

rajouté au vase. Fouilles

de laZAC de l'Enclos, 1985.

c. Moule de coupe Drag. 37

signé en lettres cursives

SANVILLI découvert lors

d'un diagnostic en 2008

dans l'atelier isolé de la Serve

d'Ervier. Il est fréquent

que des fabricants de moules

signent leur travail sous

le décor dans l'argile fraîche.

Après démoulage et ajout

du pied, cette signature

subsiste rarement.
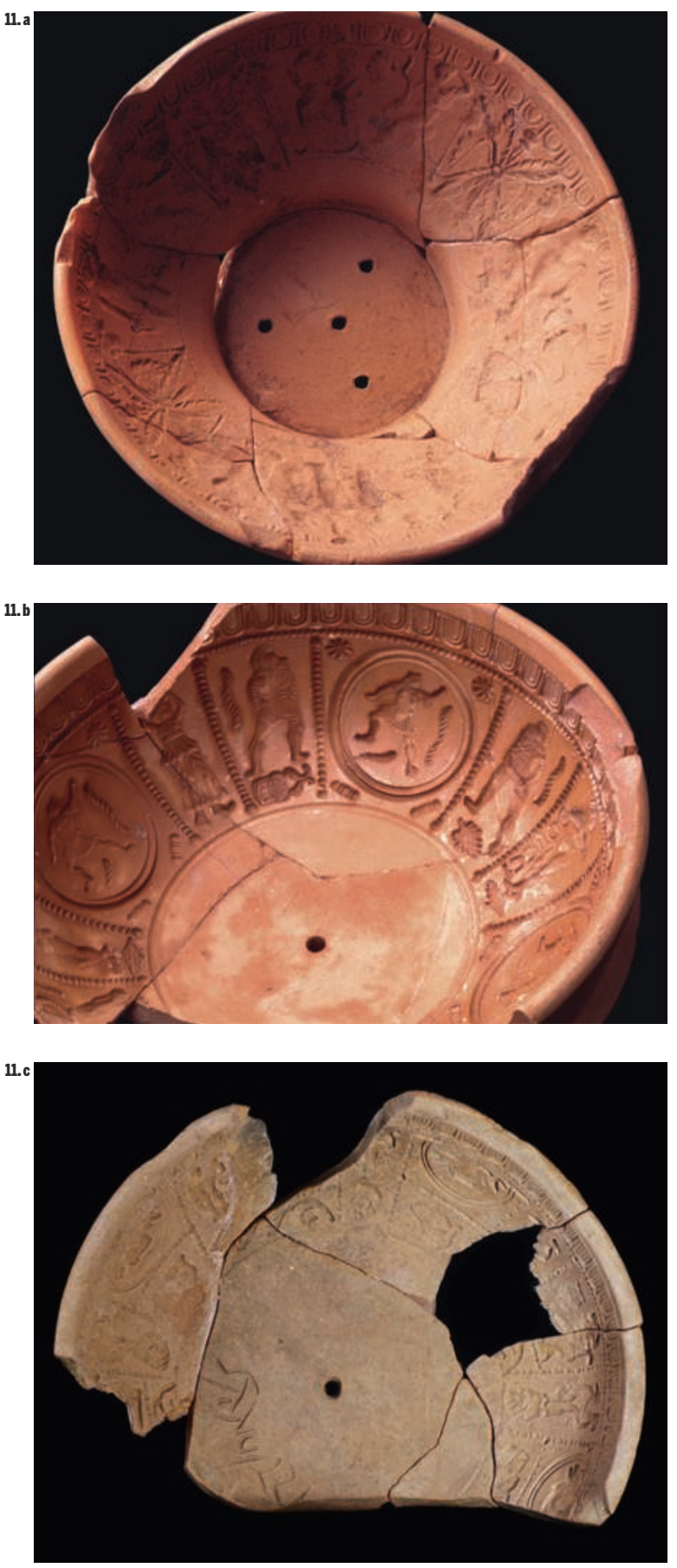

[ill. 10]. Malgré tous ces efforts et une diffusion qui dépasse le cadre de la civitas, les sigillées de Lezoux ne prévalent pas. Millau reste à la tête du marché avec une production de masse incomparable.

Mais à la fin du $\mathrm{I}^{\mathrm{er}}$ siècle ou au tout début $\mathrm{du} \mathrm{II}^{\mathrm{e}}$ siècle, la situation va totalement changer. Pour des raisons qui nous échappent, les potiers de Lezoux abandonnent leurs argiles siliceuses au profit d'une argile calcaire. Ceci fera le succès des sigillées arvernes. Le $\mathrm{II}^{\mathrm{e}}$ siècle est la période de grande exportation avec des centaines de millions de vases produits. Lezoux ne reprend cependant pas tout le marché qu'avaient conquis les ateliers du Sud de la Gaule. Sa zone d'exportation couvre largement la partie septentrionale de l'Empire romain mais s'étend très faiblement vers Burdigala, la Narbonnaise ou l'Italie et ne semble pas franchir la Méditerranée. Si Millau décline, elle continue à produire et surtout, phénomène qui va s'accentuer dans la seconde moitié du $\mathrm{II}^{\mathrm{e}}$ siècle, d'autres centres de production de sigillée apparaissent un peu partout en Gaule (les officines argonnaises prennent, déjà, de plus en plus d'importance $^{\mathbf{6}}$ ), en Espagne, en Turquie...

Des potiers lezoviens quittent les ateliers arvernes pour aller dans le Nord-Est, à Blickweiler (Sarre) par exemple. Malgré tout, l'importance de Lezoux est indéniable : près d'un millier de noms de potiers nous sont connus pour le $\mathrm{II}^{\mathrm{e}}$ siècle et le début du III $^{\mathrm{e}}$ siècle.

Les méthodes de travail se transforment pour augmenter l'efficacité et la production. Ainsi, pour les fameux vases ornés hémisphériques Drag. 37, les fabricants des moules laissaient jusqu'alors une réserve pour le pied, comme cela était déjà le cas pour les vases carénés Drag. 29 durant tout le $\mathrm{I}^{\mathrm{er}}$ siècle. Après démoulage, le tourneur devait dégrossir le pied et le retravailler. L'innovation a été de supprimer cette réserve et il suffisait alors, après démoulage, de fixer un boudin circulaire aplati sous le fond du vase pour obtenir le pied [ill. 11]. Ces moules faisaient, par ailleurs, l'objet d'un véritable commerce. Ils étaient vendus et utilisés dans tous les groupes d'ateliers de potiers de Lezoux, également dans les ateliers-satellites du Centre de la Gaule, mais aussi à Millau où l'on a retrouvé des moules de gobelets tronconiques d'origine lezovienne (Bet, Delage, Vernhet, 1994). Des formes nouvelles, promises à un long succès, sont inventées vers la fin du $\mathrm{II}^{\mathrm{e}}$ siècle. Parmi celles-ci, il y a le mortier à déversoir en forme de tête de lion (Drag. 45, Ch. 330 ${ }^{7}$ ) et dont le fond intérieur est muni d'une râpe constituée de grains de quartz. Elle devait servir à faire des préparations culinaires non pas en cuisine, mais en présence des convives. Cette forme est fabriquée durant au moins deux siècles à Lezoux et les derniers exemplaires sont cuits, en Argonne, dans un $\mathrm{V}^{\mathrm{e}}$ siècle bien avancé. Autre exemple, un gobelet (Déch. 72) qui peut être lisse, avec un décor excisé, barbotiné ou à reliefs d'applique. Certains portent même une inscription
6. Voir l'article de

Philippe Bet, Rémy

Guadagnin et Renaud

Gosselin dans ce numéro.

7. La plupart des

typologies de céramique

font référence à leur

auteur (Dragendorff,

Chenet, Déchelette...) ou

à un site archéologique

(Haltern, Lezoux...). 


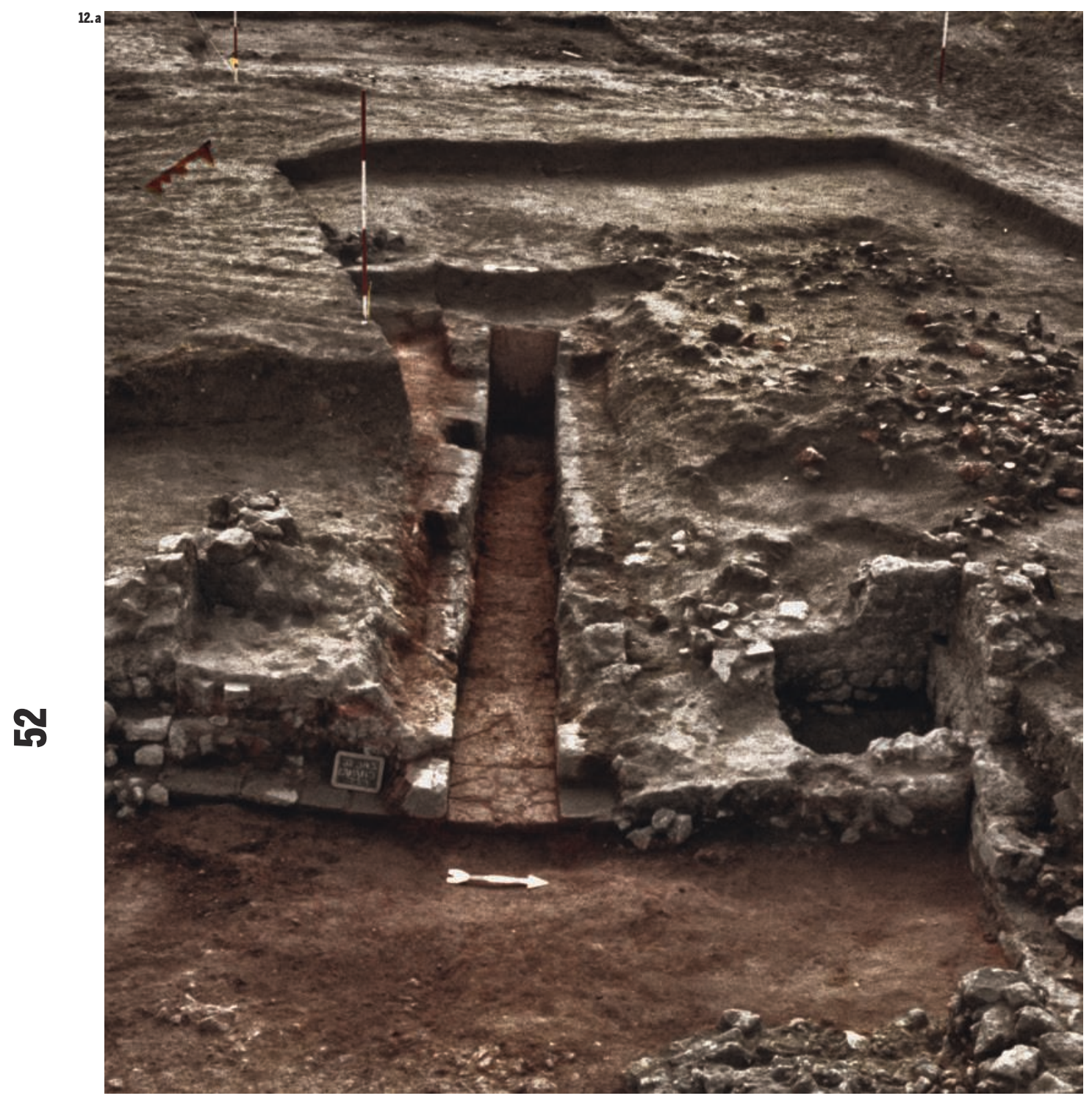

gravée avant cuisson (Bet, Delage, 1993) et font l'objet de commandes à longue distance. Ainsi, un gobelet avec une dédicace à Mithra a été découvert à Angers (Mortreau, 2013). La renommée des Arvernes et particulièrement des ateliers de Lezoux comme lieu de production d'excellence de la céramique devait être importante dans les dernières décennies du $\mathrm{II}^{\mathrm{e}}$ siècle : des artisans, comme Belsa, affichent fièrement leur appartenance à cette civitas.

Les fours à sigillée connaissent aussi de profondes transformations. Ils présentent, à la suite de l'alandier, un long canal de chauffe. Des remontées obliques de flammes partaient de là et devaient déboucher dans des tubulures qui sont beaucoup plus épaisses qu'au I ${ }^{\mathrm{er}}$ siècle. Le laboratoire de cuisson peut être cylindrique et d'un diamètre d'environ deux mètres, de plan ovale, voire rectangulaire ou carré. C'est vraisemblablement le cas du grand four de la ZAC de l'Enclos avec un canal de chauffe long de 7,20 m [ill. 12]. Aucun four à sigillée n'ayant été conservé en élévation, ni à Lezoux, ni ailleurs, la capacité d'enfournement des fours ne nous est pas connue, mais devait certainement permettre la cuisson simultanée de plusieurs milliers de vases [ill. 13].

\section{Le début d'un lent déclin}

Le III $^{\mathrm{e}}$ siècle, malgré des innovations réussies comme la mise au point de la céramique métallescente, marque le début du déclin des ateliers lezoviens. Ces difficultés sont directement liées à celles que l'Empire traverse à partir de la fin $\mathrm{du} \mathrm{II}^{\mathrm{e}}$ siècle notamment sur ses marges orientales où s'exportaient les productions de Lezoux et de Gaule centrale. Les catalogues des motifs, des systèmes décoratifs et des formes se réduisent. De vieux moules du $\mathrm{II}^{\mathrm{e}}$ siècle, ébréchés, sont retrouvés et remis en service. La conduite des fours est moins bien menée et la température de cuisson insuffisante : les vases ne sont plus rouges mais orangés. 

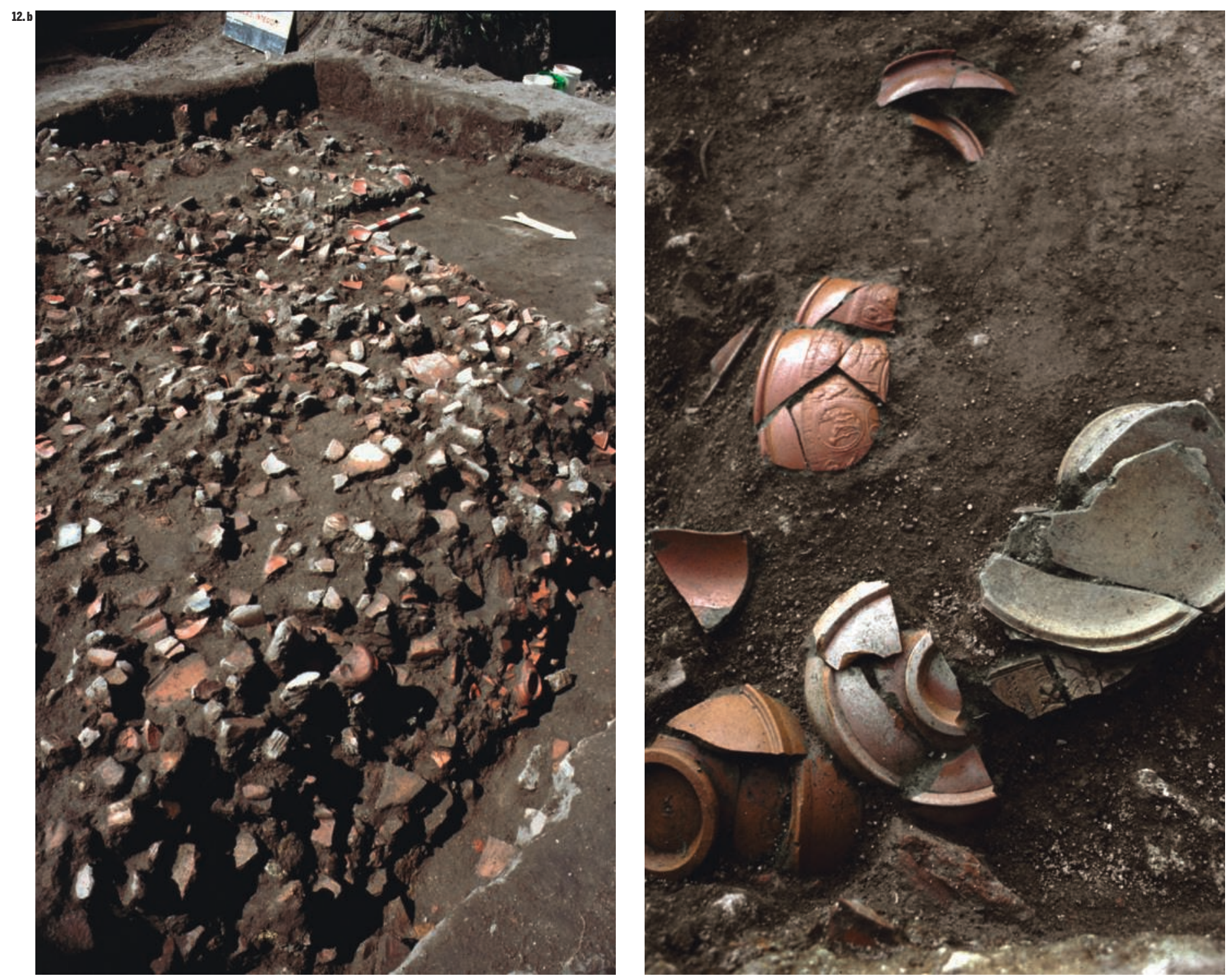

Ce qui se passe à Lezoux entre le dernier quart du III ${ }^{e}$ et le milieu du IV siècle reste peu connu. Dans la seconde moitié du IV siècle, une activité potière conséquente se maintient toujours dans le groupe des ateliers de la rue Saint-Taurin, aussi bien sur la ZAC de l'Enclos que dans le quartier de Mon Repos. Un nouveau répertoire de formes a été mis au point (Bet, Wittmann, 1995). Les motifs sont principalement repris, par surmoulage, sur des tessons de moules du $\mathrm{II}^{\mathrm{e}}$ siècle récupérés dans des tessonnières. Ce processus entraîne mécaniquement, par le phénomène de la rétraction de l'argile, une réduction de la taille du motif et une perte de qualité. Il a été employé sciemment par les potiers du $\mathrm{III}^{\mathrm{e}}$ siècle pour obtenir une réduction de la taille des têtes de lion devant orner des mortiers afin de les adapter aux différents modules de vases. Les moules du BasEmpire sont beaucoup moins soignés et, n'étant plus lissés avant l'estampage des motifs, sont marqués des stries de tournage. La diffusion dépasse toujours le cadre de la civitas et des exemplaires se retrouvent au sud chez les Vellaves et au nord-ouest, sur les rives de la Loire, chez les Turones.

Cette sigillée tardive, qui maintient dans son répertoire les dernières coupes sigillées ornées (forme Drag. 37) fabriquées dans tout l'Empire (Bet, Dousteyssier, 2014, p. 100-101), est-elle le signe de l'attachement des Arvernes à la culture antique ? La résistance de ceux-ci de 470 à 475 fait de cette cité l'un des derniers bastions romains de la Gaule que Julius Nepos préféra, finalement, livrer aux Wisigoths en échange de la Provence. Il est probable qu'en ce dernier quart du $\mathrm{v}^{\mathrm{e}}$ siècle, le dernier four à sigillée de Lezoux avait déjà été abandonné depuis longtemps, mettant un terme définitif à la technique de la sigillée moulée qui, durant tant de siècles, avait permis de diffuser des images d'un monde romain à travers de multiples contrées. 

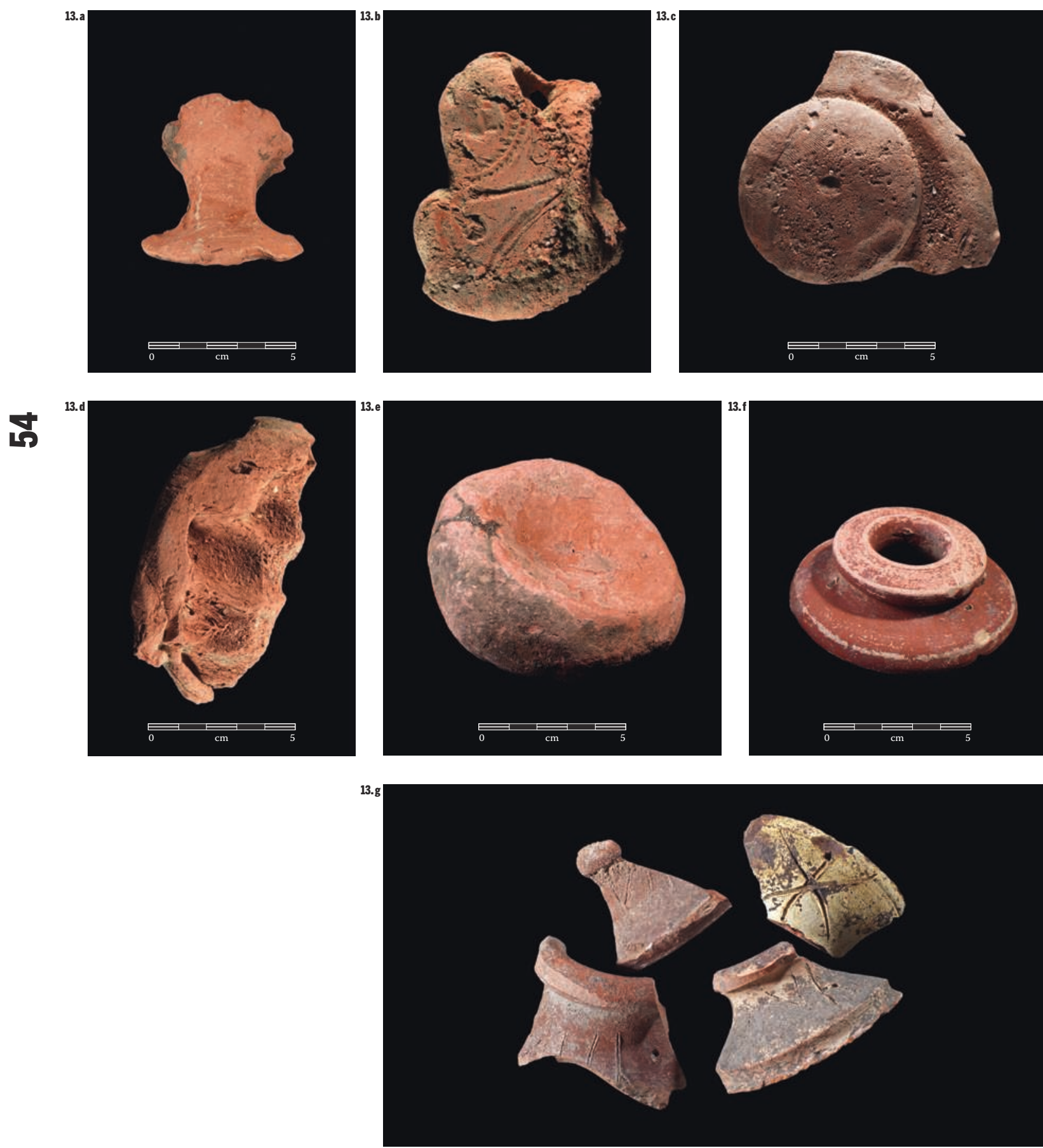

13. Ensembles de petits

éléments de calage des vases placés lors de l'enfournement (1' $1 / 2 \|^{e} s_{\text {.) }}$, issus du site Terrain Dardinier faisant partie des ateliers de la route de Maringues.

a. Ce colifichet présente

une empreinte digitale,

marque d'un façonnage

rapide au moment de

son utilisation dans une

argile à sigillée.

b. Les colifichets gardent souvent l'empreinte du décor

des vases contre lesquels

ils étaient placés.

c. Des boules d'argile fraîche, appelées massettes, sont

aussi employées pour caler les piles de vases. Celle-ci garde l'empreinte d'un pied

de vase et de traces palmaires laissées par le potier qui a calé les vases.

d. Lut de calage avec

empreintes palmaires et

traces de doigts.

e. Cette massette supportait

une pile d'assiettes

ombiliquées. L'estampille

de la première assiette s'est

imprimée sur la massette,

fraiche et malléable, donc.

au moment de son utilisation.

f. Un support tronconique

placé entre deux petites piles

de vases. Curieusement,

ils sont très souvent revêtus

d'un vernis sigillé.

g. Les supports tronconiques

portent quelquefois des

inscriptions conviviales

(AV ou AVII bonjour ;

VALE porte toi bien... ou des étoiles. 


\section{Références bibliographiques}

Baratte F., Joly M., BÉAL J.-C. (éd.), 2007, Luxe et quotidien en Gaule romaine, Mâcon, Institut de recherche du Val de Saône-Mâconnais, 245 p.

Batigne-Vallet C., Bet P., Roma V., 2016, « Production et consommation au sein du groupe des ateliers de la route de Maringues à Lezoux, l'exemple du terrain Pion ", in Actes du congrès d'Autun, 5-8 mai 2016, Marseille, Société française d'étude de la céramique antique en Gaule (SFECAG), p. 639-657.

BÉMONT C., ЈАСОВ J.-P. (dir.), La terre sigillée galloromaine, lieux de production du Haut-Empire: implantations, produits, relations, Paris, Éd. de la Maison des sciences de l'homme, Documents d'archéologie française 6, $291 \mathrm{p}$.

Béranger D., Bet P., Houdayer M., 2015, «Vaisseliers des périodes néronienne et flavienne à Lezoux », in Actes du congrès de Nyon, 14-17 mai 2015, Marseille, SFECAG, p. 435-451.

BEт P., 1988, « Groupes d'ateliers et potiers de Lezoux (Puy-de-Dôme) durant la période gallo-romaine », in Actes du congrès d'Orange, 12-15 mai 1988, Marseille, SFECAG, p. 221-241.

BET P., 1994, « Les productions céramiques antiques de Lezoux », in Provost M., Mennessier-Jouannet C. (dir.), Carte archéologique de la Gaule, le Puy-deDôme, Académie des Inscriptions et Belles-Lettres, p. 117-127.

BET P., 2012, « De l'argile à la céramique : le centre de production de Lezoux à l'époque romaine ", Géologues, n 173, p. 61-66.

BET P., 2015, « Hugues Vertet, des hommes et des pots », in Actes du congrès de Nyon, 14-17 mai 2015, Marseille, SFECAG, p. 4-12.

BET P., 2016, « Hugues Vertet, un archéologue du CNRS en Auvergne ", Bilan d'activité Auvergne 2015 Journée régionale de l'archéologie 2016, DRAC Auvergne-Rhône-Alpes, p. 91-93.

Bet P., Boudriot G., Chappet C. et Vallat P., 1998 , «Un nouveau centre de production de céramique sigillée en Gaule, le site des Queyriaux à Cournon d'Auvergne (Puy-de-Dôme) », in Actes du congrès d'Istres, 21-24 mai 1998, Marseille, SFECAG, p. 263-270.

Bet P., Chuniaud K., 1995, « Fours de potiers galloromains du site du Rincé (groupe des ateliers de Ligonnes) », Revue archéologique Sites, n 58-59, p. 35-44.

Bet P., Delage R., Murat R., 2000, « En Gaule centrale, le centre de production sigillée de Manglieu (Puy-deDôme) ", in Actes du congrès de Libourne, Marseille, SFECAG, p. 511-514.

Bet P., Delage R., Pouenat P., 2012, « Récentes fouilles sur les groupes d'ateliers de potiers de la rue SaintTaurin et de la route de Maringues à Lezoux en 2011 ", in Bilan d'activité 2010-2011 / Journée régionale de l'archéologie 2012, Direction régionale des affaires culturelles, service régional de l'Archéologie Auvergne, p. 100-102.

Bet P., Delage R., Vernhet A., 1994, «Lezoux et Millau, confrontation d'idées et de données », in Actes du congrès de Millau, 12-15 mai 1994, Marseille, SFECAG, p. 43-62.

Bet P., Delor A., 200o, « La typologie de la sigillée lisse de Lezoux et de la Gaule centrale du Haut-Empire. Révision décennale ", in Actes du congrès de Libourne, Marseille, SFECAG, p. 461-484

Bet P., Delor A., 2002, « Les premiers ateliers céramiques de type méditerranéen en Auvergne, l'exemple des officines de sigillée », in RIVET L., SCiallano M. (dir.), Vivre, produire, échanger: reflets méditerranéens, Mélanges offerts à Bernard Liou, Montagnac, M. Mergoil, « Archéologie et Histoire Romaine » 8, p. 235-242.

Bet P., Dousteyssier B. (dir.), 2014, Éclats arvernes, fragments archéologiques ( $I^{e r}-V^{e}$ siècle apr. J.-C.), Clermont-Ferrand, Presses universitaires Blaise Pascal, 333 p.
Bet P., GANGLOFf R., 1987, « Les installations de potiers gallo-romains sur le site de la ZAC de l'Enclos à Lezoux (Puy-de-Dôme) ", in Actes du congrès de Caen, 28-31 mai 1987, Marseille, SFECAG, p. 145-158.

Bet P., Gangloff R., Vertet H., 1987, Les productions céramiques antique de Lezoux et de la Gaule centrale à travers les collections archéologiques du musée de Lezoux (63), Recherches sur les ateliers de potiers de la Gaule centrale, t. IV, Avignon, $130 \mathrm{p}$.

Bet P., Gras D., 1999, « Parois fines engobées et céramique métallescente de Lezoux », in Céramiques engobées et métallescentes gallo-romaines, Oxford, Rei Cretariæ Romanæ Fautorum Acta, Supplementum 8, p. 13-38.

Bet P., Montineri D., 1989, «La céramique sigillée moulée tibéro-claudienne du site de la ZAC de l'Enclos à Lezoux », in Actes du congrès de Lezoux, 4-7 mai 1989, Marseille, SFECAG, p. 55-69.

Bet P., Valaude P., Vertet H., 1986, «Le centre de production de Courpière ", in BÉMONT, JАCOB, 1986, p. 144-145

Bet P., Vertet H., 1986, « Le centre de production de Lezoux », in BÉmont, ЈАCOB, 1986, p. 139-145.

Bet P., Wittmann A., 1995, « La production de la céramique sigillée à Lezoux (Auvergne, France) durant le Bas-Empire », Rei Cretarice Romance Fautorum Acta, $\mathrm{n}^{\circ}$ 34, Alba Regia, Székesfehérvar (Hongrie), p. 205-220, pl. XV-XVIII.

Chuniaud K., 2002, « Le groupe des ateliers de potiers de Ligonnes à Lezoux (Puy-de-Dôme) : un champ d'étude pour les questions relatives à l'organisation de la production céramique en Gaule romaine », in Rivet L., Sciallano M. (dir.), Vivre, produire, échanger : reflets méditerranéens, Mélanges offerts à Bernard Liou, Montagnac, M. Mergoil, «Archéologie et Histoire Romaine » 8, p. 243-24.8.

Corrocher J., 1994, Le site de Lubillet, ancienne paroisse de Lapalisse, Vichy, Centre de recherches archéologiques et historiques de Vichy et de sa région, $64 \mathrm{p}$

DESBAT A., 1993, « Observations sur les fours à tubulures des $\mathrm{I}^{\mathrm{er}}$ et $\mathrm{II}^{\mathrm{e}}$ siècles à Lezoux ", in Actes du congrès de Versailles, 20-23 mai 1993, Marseille, SFECAG, p. 361-368

Desbat A., Genin M., Lasfargues J., 1996, Les productions des ateliers de potiers antiques de Lyon, $1^{\text {re }}$ partie: Les ateliers précoces, Gallia, 53, $365 \mathrm{p}$.

Dousteyssier B., 2011, La cité des Arvernes, $I^{e r}-I^{e}$ siècles apr. J.-C., Clermont-Ferrand, Lemme édit., «Illustoria » HA 13, $118 \mathrm{p}$.

Dousteyssier B., Segard M., Trément F., 2004, «Les villae gallo-romaines dans le territoire proche d'Augustonemetum - Clermont-Ferrand ", Revue archéologique du Centre de la France, t. 43, http: //racf.revues.org/143

FÉrAudy L. DE, VERTET H., 1986, « Le centre de production de Toulon-sur-Allier/Le Lary ", in BÉMONT, ЈАCOB, 1986, p. 153-155.

FERDIÈrE A., 2014, «Un site de production remarquable à Lezoux : Le Teix/Les Fromentaux", in Bet, DOusTeYsSIER, 2014, p. 204-205.

Genin M., SchaAd D., 2007, La Graufesenque (Millau, Aveyron), Pessac, Éd. de la Fédération Aquitania, vol. I, Condatomagos, une agglomération de confluent en territoire rutène. II ${ }^{e}$ s. a. C.-III s. p. C. SChaAd D. (dir.), 378 p. ; vol. II, Sigillées lisses et autres productions, GENIN M. (dir.), $589 \mathrm{p}$.

HaAlebos J.-K., 1977, Ars Cretaria. Nijmegen en La Graufesenque, Nijmegen, Libelli Noviomagenses, 4, $40 \mathrm{p}$.

HenRY M.-J., 2014, « D'une industrie de prestige à l'autre : la Serve d'Ervier », in Bet, Dousteyssier, 2014, p. 74-75.

JoUANAUD J.-L., 1994, « Les timbres sur tuiles de Théodoric et Athalaric », in Actes du congrès de Millau, 12-15 mai 1994, Marseille, SFECAG, p. $183-188$.
Lutz M., 1977, « Cheminement d'un style de la Gaule du Sud à la Gaule de l'Est », Revue archéologique $d u$ Centre de la France, t. 16, fasc. 1-2, p. 107-116.

Menez Y., 1989, « Les céramiques fumigées ("Terra Nigra") du Bourbonnais. Étude des collections de Néris-les-Bains et Châteaumeillant ", Revue archéologique du Centre de la France, t. 28, fasc. 2, p. 117-178.

Mennessier-Jounnnet C., 1991, « Un four de potier de La Tène Finale à Lezoux (Puy-de-Dôme) », Revue archéologique du Centre de la France, vol. 30 , fasc. 1, p. 113-126.

Mortreau M., 2013, « Les lampes plastiques et le luminaire à tête de Nubien du mithraeum d'Angers ", Archéopages, 36, Exotismes, p. 16-17.

Notet J.-C., 2012, La production de sigillée et de céramique fine gallo-romaine de Gueugnon (Saône-et-Loire), Revue archéologique de l'Est, $32^{\mathrm{e}}$ suppl., $256 \mathrm{p}$.

Piboule A., Sénéchal R., Vertet H., 1981, Les potiers de Lezoux du premier siècle: Titos, Revue archéologique Sites, hors-série n ${ }^{\circ} 8,144 \mathrm{p}$

PICON M., 1973, Introduction à l'étude technique des céramiques sigillées de Lezoux, Dijon, Centre de recherche sur les techniques grécoromaines, $135 \mathrm{p}$.

PiCON M., 2002, « Les modes de cuisson, les pâtes et les vernis de la Graufesenque : une mise au point ", in Genin M., Vernhet A. (dir.), Céramiques de la Graufesenque et autres productions d'époque romaine. Nouvelles recherches. Hommages à Bettina Hoffmann, Montagnac, M. Mergoil, « Archéologie et Histoire Romaine » 7, p. 139-163.

Rogers G.-B., 1974, Poteries sigillées de la Gaule centrale, 1. Les motifs non figurés, Gallia, suppl. 28, Paris, Éditions du Centre national de la recherche scientifique, $196 \mathrm{p}$.

Rogers G.-B., 1999, Poteries sigillées de la Gaule centrale, les potiers, Lezoux, Laboratoire de céramologie antique de Lezoux, «Cahier du Centre archéologique de Lezoux » 1, 2 vol., 505 p.

RoMEuF A.-M., 200o, Le quartier artisanal gallo-romain des Martres-de-Veyre (Puy-de-Dôme), Lezoux, Laboratoire de céramologie antique de Lezoux, "Les cahiers du Centre archéologique de Lezoux » 2, 2 vol., $258+458$ p.

Stanfield J. A., Simpson G., 1990, Recherches sur les ateliers de potiers de la Gaule centrale. 5 Les potiers de la Gaule centrale, Gonfaron, Revue archéologique Sites, hors-série $n^{\circ} 37,450 \mathrm{p}$.

TourGON D., 2014, « Un vase à reliefs d'applique fabriqué en territoire arverne : le Décheletyte 74 ", in Bet, Dousteyssier, 2014, p. 86-87.

VAUTHEY M., 1993, L'atelier de potiers gallo-romains de Terre-Franche-sur-Allier, Gonfaron, Revue archéologique Sites, hors-série n ${ }^{\circ} 39,100$ p.

VerTET H., 1967, « Céramique sigillée tibérienne à Lezoux », Revue archéologique, 2, p. 255-286.

Vertet H., 1980, « Carte des ateliers de potiers de la Gaule centrale ", Recherches sur les ateliers de potiers de la Gaule centrale, Revue archéologique Sites, hors-série n ${ }^{\circ}$ 6, p. 13-41.

VERTET H., 1990, « Observations sur la sociologie et l'économie des ateliers de potiers gallo-romains du Centre de la Gaule », Bulletin de la Société Nationale des Antiquaires de France, p. 127-136.

Vertet H., Bet P., 1980, « Fouille du terrain de l'CEuvre Grancher, les structures du second siècle, à Lezoux ", Recherches sur les ateliers de potiers de la Gaule centrale, Revue archéologique Sites, hors-série $n^{\circ} 6$, p. 43-72. 